\title{
Observer Design for a Class of Nonlinear Discrete Time Systems: Real Time Application to the One-link Flexible Joint Robot
}

\author{
Assem Thabet \\ Laboratoire de Recherche MACS, ENIG \\ University of Gabes, Tunisia
}

\author{
Noussaiba Gasmi \\ Centre de Recherche en Automatique de Nancy \\ CRAN-CNRS UMR 7039 \\ University of Lorraine, FRANCE \\ Laboratoire de Recherche MACS, ENIG \\ University of Gabes, Tunisia
}

\begin{abstract}
This paper demonstrates the observer design for large class of nonlinear discrete time systems. The use of the differential mean value theorem (DMVT) allows transforming the nonlinear error dynamics into a linear parameter varying (LPV) system. This has the advantage of introducing a general condition on the nonlinear functions. To ensure asymptotic stability, sufficient conditions are expressed in terms of linear matrix inequalities (LMIs). For comparison, an observer based on the use of the one-sided Lipschitz condition is introduced. High performances are shown through real time implementation of the one-link flexible joint robot to ARDUINO MEGA 2560 device.
\end{abstract}

\section{General Terms}

Nonlinear Observers, ARDUINO MEGA 2560

\section{Keywords}

Discrete time systems, DMVT, One-sided Lipschitz condition, Quadratic inner-boundedness, LMIs

\section{INTRODUCTION}

The design of observer for nonlinear systems satisfying a Lipschitz continuity condition have been important topics in nonlinear system theory for over three decades, resulting in a substantial amount of literature; see [8]-[17]-[9]-[3]-[1]-[7] and the references inside.

The most existing results concern continuous time systems with few extensions to discrete-time ones [10]. As no universal approach exists, state observers, in particular for nonlinear systems, are still a challenging and open problem. Beside the famous extended Kalman filter [14] [4] [5] (and its real time application with DSP device [15]), we distinguish a simple and useful nonlinear state observer based on the solution of a Riccati-like equation and the Lipschitz condition [12] [20] [11].
Stability analysis is based on the convergence of the estimation error. This has been studied by using both Lyapunov functions and functionals where stability conditions are expressed using Linear matrix inequalities (LMIs). However, for large values of the Lipschitz constant, the stability conditions seem difficult to be satisfied [19] [18].

The basic idea of this work is to use the differential mean value theorem (DMVT) which allows writing the dynamics of the estimation error using the nonlinear function as a class of Linear parameter varying (LPV) systems [18]. Stability of the estimation error is analyzed using the convexity principle and the Lyapunov stability theory. The observer convergence of the proposed scheme is computed by LMI. The idea behind the DMVT is to provide a non restrictive sufficient conditions on nonlinear function. The aim is to ensure asymptotic convergence for a class of nonlinear discretetime systems.

This work is organized as follows. In Section 2, we introduce the problem formulation. Next, the synthesis method of the observer gain will be detailed. This method consists in LMIs feasibility conditions. The last section is devoted to the performance of the proposed approach through a real time implementation (with ARDUINO MEGA 2560 device) with a comparison between the works of [19] and [3].

Notations : The following notations will be used throughout this paper.

- $A^{T}$ represents the transposed matrix of $A$;

- For a square matrix $S, S>0(S<0)$ means that this matrix is positive definite (negative definite) ;

- The set $C o(x, y)=\{\lambda x+(1-\lambda) y, 0 \leq \lambda \leq 1\}$ is the convex hull of $x, y$;

- $e_{s}(i)=(\underbrace{0, \ldots, 0, \overbrace{1}^{i^{t h}}, 0, \ldots, 0}_{s-\text { components }})^{T} \in \mathbb{R}^{s}, s \geq 1$, is a vector of the canonical basis of $\mathbb{R}^{s}$; 
- In a matrix, the notation $(\star)$ is used for the blocks induced by symmetry ;

- $<x, y>=x^{T} y$ is the scalar product ;

- $\|x\|=\sqrt{\langle x, x\rangle}=\sqrt{x^{T} x}$ is the Euclidean vector norm.

\section{PROBLEM STATEMENT}

Consider the class of nonlinear systems described by the following set of equations :

$$
\left\{\begin{array}{l}
x(k+1)=A x(k)+f(x(k), u(k)) \\
y(k)=C x(k)
\end{array}\right.
$$

where $x(k) \in \mathbb{R}^{n}, u(k) \in \mathbb{R}^{m}$ and $y(k) \in \mathbb{R}^{p}$ denote respectively the state, the input and the linear output. $\mathrm{A}$ and $\mathrm{C}$ are constant matrices of adequate dimensions. $f: \mathbb{R}^{n} \times \mathbb{R}^{m} \longrightarrow \mathbb{R}^{n}$ is a real nonlinear vector field.

We consider now the standard state observer :

$$
\left\{\begin{array}{l}
\hat{x}(k+1)=A \hat{x}(k)+f(\hat{x}(k), u(k))+L(y(k)-\hat{y}(k)) \\
\hat{y}(k)=C \hat{x}(k)
\end{array}\right.
$$

where $\hat{x}(k)$ denotes the estimate of the state $x(k)$.

The estimation problem consists in determining a gain $L$ where the estimation error $\varepsilon(k)=x(k)-\hat{x}(k)$ converges asymptotically to zero. The dynamic of the estimation error is expressed as follows :

$$
\varepsilon(k+1)=(A-L C) \varepsilon(k)+\Delta f(k)
$$

where $\Delta f_{k}=f(x(k), u(k))-f(\hat{x}(k), u(k))$.

Note that all the approaches developed by now attempt to dominate the term $\Delta f_{k}$ by using directly the Lipschitz property. In the next section, we will present a recent method given by $|3|$ based on onesided Lipschitz condition.

\subsection{One-sided Lipschitz Observer}

The observer synthesis [3] [1] is based on the following two assumptions :

\section{- Assumption 1}

fis one-sided Lipschitz with respect to $x(k)$.i.e,

$$
\langle f(x, u)-f(\hat{x}, u), x-\hat{x}\rangle \leq \rho\|x-\hat{x}\|^{2}
$$

for any $x, \hat{x} \in \mathbb{R}^{n} ; u \in \mathbb{R}^{m} ; y \in \mathbb{R}^{p}$

where $\rho$ is the so-called one-sided Lipschitz constant which can be positive or negative.

\section{- Assumption 2}

$f$ is quadratically inner-bounded with respect to $x(k)$.i.e,

$$
\begin{aligned}
& \|f(x, u, y)-f(\hat{x}, u)\|^{2} \leq \beta\|x-\hat{x}\|^{2} \\
& +\gamma\langle x-\hat{x}, f(x, u)-f(\hat{x}, u)\rangle
\end{aligned}
$$

where $\beta$ and $\gamma$ are real scalars.

Unlike the well-known Lipschitz condition, the constants $\rho, \beta$ and $\gamma$ can be positive, negative or zero. In addition, if the function $f$ is Lipschitz, then it is also both one-sided Lipschitz and quadratically inner-bounded $(\beta>0$ and $\gamma)$. The concept of quadratic innerboundedness (5), is very useful to provide tractable LMI stability conditions.

Using the above assumption, the following theorem provides sufficient conditions so that (2) is an asymptotic full-order observer for system 1 .
THEOREM 1. Under Assumption 1, system (2) is an asymptotic observer for system (1) if there exist scalars $\alpha>0, \mu_{1}>0, \mu_{2}>$ $0, \rho, \beta, \gamma$ and $\epsilon>0$ and matrices $P=P^{T}>\alpha I_{n}, Q=Q^{T}>0$, $S$ and $X$ that solve the following $L M I$ :

$$
\left[\begin{array}{cc}
P & S \\
S^{T} & Q
\end{array}\right]>0
$$

and

$$
\mathbb{N}<0
$$

where $\mathbb{N}$ is given by :

$$
v \mathbb{N}=\left[\begin{array}{cccccc}
N_{11} & N_{12} & 0 & N_{14} & N_{14} & 0 \\
\star & N_{22} & N_{23} & 0 & 0 & 0 \\
\star & \star & N_{33} & 0 & 0 & N_{23}^{T} \\
\star & \star & \star & -\eta^{-1} P & 0 & 0 \\
\star & \star & \star & \star & -\epsilon I_{n} & 0 \\
\star & \star & \star & \star & \star & -\epsilon^{-1} \alpha^{2} I_{n}
\end{array}\right]
$$

with

$$
\left\{\begin{array}{l}
\eta=1+2(|\beta|+|\rho|) \\
\mathbb{S}=S-\left(\mu_{1} \gamma-\mu_{2}\right) I_{n} \\
N_{11}=-P+2\left(\mu_{1} \beta+\mu_{2} \rho\right) I_{n} \\
N_{12}=\eta A^{T} P-\eta C^{T} X-\mathbb{S} \\
N_{14}=A^{T} P-C^{T} X \\
N_{22}=\eta P-Q-2 \mu_{1} I_{n} \\
N_{23}=S+\alpha(\gamma-1) I_{n} \\
N_{33}=Q-2 \alpha I_{n} .
\end{array}\right.
$$

Then, the gain for observer is given by $L=P^{-1} X^{T}$.

PROOF. In this section we present some guiding steps. Let us consider the quadratic Lyapunov function :

$$
V(k)=\left[\begin{array}{c}
\varepsilon(k) \\
\Delta f_{k}
\end{array}\right]^{T}\left[\begin{array}{cc}
P & S \\
S^{T} & Q
\end{array}\right]\left[\begin{array}{c}
\varepsilon(k) \\
\Delta f_{k}
\end{array}\right]
$$

The variation $\Delta V=V(k+1)-V(k)$ of this Lyapunov function is given by:

$$
\begin{aligned}
\Delta V= & \varepsilon^{T}(k+1) P \varepsilon(k+1)-\varepsilon^{T}(k) P \varepsilon(k)-\Delta f_{k}^{T} Q \Delta f_{k} \\
& +\Delta f_{k+1}^{T} Q \Delta f_{k+1}+2 \varepsilon^{T}(k+1) S \Delta f_{k+1}-2 \varepsilon^{T}(k) S \Delta f_{k}
\end{aligned}
$$

The one-sided Lipschitz and the quadratically inner-bounded conditions (4) and (5) give the following inequality :

$$
\left\{\begin{array}{l}
\mu_{2} \rho \varepsilon^{T}(k) \varepsilon(k)-\mu_{2} \varepsilon^{T}(k) \Delta f_{k} \geq 0 \\
\mu_{1} \beta \varepsilon^{T}(k) \varepsilon(k)+\mu_{1} \gamma \varepsilon^{T}(k) \Delta f_{k}-\mu_{1} \Delta f_{k}^{T} \Delta f_{k} \geq 0
\end{array}\right.
$$

where $\mu_{1}$ and $\mu_{2}$ are arbitrary strictly positive scalars.

With the fact that $P>\alpha I_{n}$ and using (12) in (11), it follows that :

$$
\begin{aligned}
\Delta V \leq & \eta \varepsilon^{T}(k+1) P \varepsilon(k+1)+\varepsilon^{T}(k)\left(-P+2\left(\mu_{2} \rho+\mu_{1} \beta\right) I\right) \varepsilon(k) \\
& -\Delta f_{k}^{T}\left(Q+2 \mu_{1} I\right) \Delta f_{k}-2 \varepsilon^{T}(k)\left(S+\left(\mu_{2}-\mu_{1} \gamma\right) I\right) \Delta f_{k} \\
& +2 \varepsilon^{T}(k+1)(S+\alpha(\gamma-1) I) \Delta f_{k+1} \\
& +\Delta f_{k+1}^{T}(Q-2 \alpha I) \Delta f_{k+1}
\end{aligned}
$$

On the other hand, using the dynamics of the estimation error (3) and based on the Lyapunov stablity theory, the convergence of the estimation error is guaranteed, as soon as $\Delta V<0$ is negative definite, which holds true if

$$
\chi^{T} \mathfrak{N} \chi<0
$$

where

$$
? \chi^{T}(k)=\left[\begin{array}{lll}
\varepsilon^{T}(k) & \Delta f_{k}^{T} & \Delta f_{k+1}^{T}
\end{array}\right]
$$


and

$$
\mathfrak{N}=\left[\begin{array}{ccc}
N_{11}+\eta \mathcal{N}_{12} P^{-1} \mathcal{N}_{12}^{T} & \eta \mathcal{N}_{12}-\mathbb{S} & \mathcal{N}_{12} P^{-1} N_{23} \\
\star & N_{22} & N_{23} \\
\star & \star & N_{33}
\end{array}\right]
$$

with $\mathcal{N}_{12}=(A-L C)^{T} P$

The BMI problem of (14)-(16) is not convex (the term $T_{1}=$ $\mathcal{N}_{12} P^{-1} N_{23}$ ). To linearize this problem, [3] proposes a three-step procedure. This procedure is based on rewriting $[16$ as :

$$
\mathfrak{N}=\overline{\mathfrak{N}}+\psi \phi^{T}+\phi \psi^{T}
$$

where $\overline{\mathfrak{N}}$ is a linear part.

$\psi \phi^{T}+\phi \psi^{T}$ will compensate the term $T_{1}$ with $\psi^{T}=\left[\begin{array}{lll}\mathcal{N}_{12}^{T} & 0 & 0\end{array}\right]$ and $\phi^{T}=\left[\begin{array}{lll}0 & 0 & P^{-1} N_{23}\end{array}\right]$.

Now, using the well-known matrix inequality :

$$
\psi \phi^{T}+\phi \psi^{T} \leq \epsilon \phi \phi^{T}+\frac{1}{\epsilon} \psi \psi^{T}
$$

with $\epsilon>0$.

The BMI can be linearized to give the LMI 77. All the details and procedures are given in [3].

The subject of the next section is to exploit the Lipschitz condition to obtain non restrictive synthesis conditions.

\subsection{Observer based on DMVT}

This section is dedicated to present some steps to the proposed approach. First, we assume that the Jacobian matrix of $f$ satisfies the following condition [19] :

$$
a_{i j} \leq \frac{\partial f_{i}(x, u, y)}{\partial x_{j}} \leq b_{i j}
$$

where

$$
\begin{aligned}
& a_{i j}=\min _{Z \in \mathbb{R}^{n} \times \mathbb{R}^{m} \times \mathbb{R}^{p}}\left(\frac{\partial f_{i}}{\partial x_{j}}(Z)\right) \\
& b_{i j}=\max _{Z \in \mathbb{R}^{n} \times \mathbb{R}^{m} \times \mathbb{R}^{p}}\left(\frac{\partial f_{i}}{\partial x_{j}}(Z)\right)
\end{aligned}
$$

Now, we present some synthesis conditions to ensure asymptotic convergence, in particular, we provide a non-restrictive suf?cient condition to assure the feasibility of a LMI [2], easily tractable by convex optimization algorithms.

First, we need to define the set $\mathcal{H}$ as follows :

$$
\begin{aligned}
\mathcal{H}_{n, n}= & \left\{v=\left(v_{11}, \ldots, v_{1 n}, \ldots, v_{n n}\right)\right. \\
& \left.: a_{i j} \leq v_{i j} \leq b_{i j}, i=1, \ldots, n ; j=1, \ldots, n\right\}
\end{aligned}
$$

The set $\mathcal{H}_{n, n}$ is a bounded convex domain of which the set of vertices is defined by :

$$
\mathcal{V}_{\mathcal{H}_{n, n}}=\left\{\alpha=\left(\alpha_{11}, \ldots, \alpha_{1 n}, \ldots \alpha_{n n}\right): \alpha_{i j} \in\left\{a_{i j}, b_{i j}\right\}\right\}
$$

Secondly, the affine matrix function is given by :

$$
\Upsilon(v)=A+\sum_{i, j=1}^{n, n} v_{i j} e_{n}(i) e_{n}^{T}(j)
$$

where : $v \in \mathcal{H}_{n, n}$.

Now, state the theorem below to synthesis a useful observer for Lipschitz nonlinear systems.
THEOREM 2. The estimation error $\varepsilon(k)$ is asymptotically stable if there exist matrices $P=P^{T}>0$ and $R$ of appropriate dimensions such that the following LMI is feasible :

$$
\begin{gathered}
\text { Block }-\operatorname{diag}\left(\Gamma\left(\alpha^{1}\right), \Gamma\left(\alpha^{2}\right), \ldots, \Gamma\left(\alpha^{2^{n n}}\right)\right)<0, \\
\alpha^{j} \in \mathcal{V}_{\mathcal{H}_{n, n}} ; \text { for } j=1, \ldots, 2^{n n} .
\end{gathered}
$$

where

$$
\Gamma\left(\alpha^{j}\right)=\left[\begin{array}{cc}
-P & \Upsilon^{T}\left(\alpha^{j}\right) P-C^{T} R \\
\star & -P
\end{array}\right]
$$

Then, the gain observer is $L=P^{-1} R^{T}$.

Proof. Now, Considering the proposition below :

Proposition. (The DMVT for vector valued function [18]). Let $\varphi$ : $\mathbb{R}^{n} \rightarrow \mathbb{R}^{n}$. Let $a, b \in \mathbb{R}^{n}$. We assume that $\varphi$ is differentiable on $C o(a, b)$. Then, there are constant vectors $z_{1}, \ldots, z_{n} \in C o(a, b)$, $z_{i} \neq a, z_{i} \neq b$ for $i=1, \ldots, n$ such that

$$
\varphi(a)-\varphi(b)=\left(\sum_{i, j=1}^{n, n} e_{n}(i) e_{n}^{T}(j) \frac{\partial \varphi_{i}}{\partial x_{j}}\left(z_{i}\right)\right)(a-b) .
$$

In analogy with the approach of [3] [19] [18], and by applying the Proposition on the function $f$, we deduce that there exist $z_{i} \in$ $C o(x, \hat{x})$, for all $i=1, \ldots, n$, as follows :

$$
\begin{aligned}
\Delta f_{k} & =f(x, u, y)-f(\hat{x}, u, \hat{y}) \\
& =\left(\sum_{i, j=1}^{n, n} e_{n}(i) e_{n}^{T}(j) \frac{\partial f_{i}}{\partial x_{j}}\left(z_{i}, u, y\right)\right) \varepsilon(k)
\end{aligned}
$$

For simplicity, we consider the notation

$$
\Xi_{z_{i}}=\sum_{i, j=1}^{n, n} e_{n}(i) e_{n}^{T}(j) \frac{\partial f_{i}}{\partial x_{j}}\left(z_{i}, u, y\right)
$$

and

$$
\mathfrak{w}_{i j}(k)=\frac{\partial f_{i}}{\partial x_{j}}\left(z_{i}(k), u(k), y(k)\right)
$$

with

$$
\mathfrak{w}(k)=\left(\mathfrak{w}_{11}(k), . ., \mathfrak{w}_{1 n}(k), \ldots \mathfrak{w}_{n n}(k)\right)
$$

From (24) and 28), the dynamic of the global estimation error (3) becomes

$$
\varepsilon(k+1)=(\Upsilon(\mathfrak{w}(k))-L C) \varepsilon(k)
$$

where $\Upsilon(\mathfrak{w}(k))=A+\Xi_{z_{i}}$.

Then, the observer design problem of the class of nonlinear systems (2) is transformed to the problem of stability of a class of LPV systems 32 .

Let us consider the standard Lyapunov function :

$$
V(k)=\varepsilon(k)^{T} P \varepsilon(k)
$$

where $P=P^{T}$ is a positive-definite matrix.

The variation of this function is

$$
\begin{aligned}
\Delta V & =V(k+1)-V(k) \\
& =\varepsilon^{T}(k)\left\{(\Upsilon(\mathfrak{w}(k))-L C)^{T} P(\Upsilon(\mathfrak{w}(k))-L C)-P\right\} \varepsilon(k)
\end{aligned}
$$

Using the Schur complement [6] and the notation $R=L^{T} P$, we deduce that $\Delta V<0$ for all $\varepsilon(k) \neq 0$ if

$$
\mathcal{F}:=\left[\begin{array}{cc}
-P & \Upsilon^{T}(\mathfrak{w}(k)) P-C^{T} R \\
\star & -P
\end{array}\right]<0
$$


for all $\mathfrak{w}(k) \in \mathcal{H}_{n, n}$.

We deduce that $\Delta V<0$ if $\mathcal{F}$ is negative-definite on $\mathcal{V}_{\mathcal{H}_{n, n}}$. Since $P$ is positive-definite, then we can compute the gain $L$ as $P^{-1} R^{T}$.

\section{SIMULATION RESULTS}

Studies are carried out on the one-link flexible joint robot [7]-[13] to evaluate the performance of the proposed observers.

$$
\left\{\begin{array}{l}
\dot{\theta}_{m}=\omega_{m} \\
\dot{\omega}_{m}=\frac{k}{J_{m}}\left(\theta_{l}-\theta_{m}\right)-\frac{b}{J_{m}} \omega_{m}+\frac{K_{T}}{J_{m}} u \\
\dot{\theta}_{l}=\omega_{l} \\
\dot{\omega}_{l}=\frac{k}{J_{l}}\left(\theta_{l}-\theta_{m}\right)-\frac{m g h}{J_{l}} \sin \left(\theta_{l}\right)
\end{array}\right.
$$

where $\theta_{m}$ and $\theta_{l}$ are, respectively, the angles of rotations of the motor and link. $\omega_{m}$ and $\omega_{l}$ are their angular velocities. $J_{m}$ and $J_{l}$ are, respectively, the inertia of the motor and link. $K_{T}, k, m, g$ and $h$ are positive constants.

This system can be described by these nonlinear continuous equations :

$$
\left\{\begin{array}{l}
\dot{x}(t)=A_{c} x(t)+B_{c} u(t)+f_{c}(x(t)) \\
y(t)=C_{c} x(t)
\end{array}\right.
$$

where:

$$
\begin{aligned}
x= & {\left[\begin{array}{llll}
\theta_{m} & \omega_{m} & \theta_{l} & \omega_{l}
\end{array}\right]^{T} ; } \\
A_{c} & =\left[\begin{array}{cccc}
-10 & 1 & 0 & 0 \\
-48.6 & -1.26 & 48.6 & 0 \\
0 & 0 & -22 & 1 \\
1.95 & 0 & -19.5 & -6
\end{array}\right] ; B_{c}=\left[\begin{array}{c}
1 \\
0 \\
2 \\
0.5
\end{array}\right] ; \\
C_{c} & =\left[\begin{array}{llll}
1 & 0 & 0 & 0 \\
0 & 1 & 0 & 0
\end{array}\right] ;
\end{aligned}
$$

The nonlinear function : $f_{c}(x(t))=\left[\begin{array}{c}0 \\ 0 \\ 0 \\ \lambda \sin \left(x_{3}\right)\end{array}\right]$

with $\lambda \in \mathbb{R}$.

After the discretization (with Euler method), the discrete time systems becomes :

$$
\left\{\begin{array}{l}
x(k+1)=A x(k)+B u(k)+g(x(k)) \\
y(k)=C x(k)
\end{array}\right.
$$

where $A=I_{4}+T_{e} A_{c}, B=T_{e} B_{c}, C=C_{c}$ et $g(x(k))=$ $T_{e} g_{c}(x(t))$.

with $T_{e}=0.01 \mathrm{~s}$.

Using THEOREM 1 it is easy to verify that $f(x(k), y(k))$ satisfies condition (4) with one-sided Lipschitz constant $\rho=\lambda T_{e}$. In addition, $f(x(k))$ is a Lipschitz function, and is also quadratically inner-bounded with $\beta=\lambda T_{e}$ and $\gamma=0$.

Applying THEOREM we obtain $\mathfrak{w}_{i j}=0$ for all $(i, j) \neq(4,3)$ and $\mathfrak{w}_{43}=\lambda T_{e} \cos \left(z_{3}(k)\right)$. Then, the set of vertices $\mathcal{V}_{\mathcal{H}_{4,4}}$ can be reduced to $\mathcal{V}_{\mathcal{H}_{4,4}}=\left\{-\lambda T_{e},+\lambda T_{e}\right\}$.

In the next section, we note :

- $\mathbf{L}_{1}$ : observer gain matrix using the property of one sidedLipschitz (O.S.L).
- $\mathbf{L}_{2}$ :observer gain matrix using the DMVT theorem.

For comparison, we introduce two values of $\lambda$. First, with $\lambda=3$ and by solving LMIs of THEOREM 1 and THEOREM 2 we obtain

$$
L_{1}=\left[\begin{array}{cc}
0.5813 & 0.01 \\
-0.486 & 0.9132 \\
0 & 0.2357 \\
0.0195 & -0.1916
\end{array}\right] ; L_{2}=\left[\begin{array}{cc}
0.9 & 0.01 \\
0.486 & 1.1318 \\
0 & 0.2365 \\
0.0195 & -0.1517
\end{array}\right]
$$

The initial conditions for the system and for the observers have been chosen as: $x(0)=\left[\begin{array}{llll}0.5 & 0.5 & 0.5 & 0.5\end{array}\right]^{T}$,

$\hat{x}(0)=\left[\begin{array}{llll}-0.5 & -0.5 & -0.5 & -0.5\end{array}\right]^{T}$.

For the real time implementation on the "Arduino MEGA 2560" card, we have chosen two modes of application. The diagram illustrating the implementation is given by Figure 1

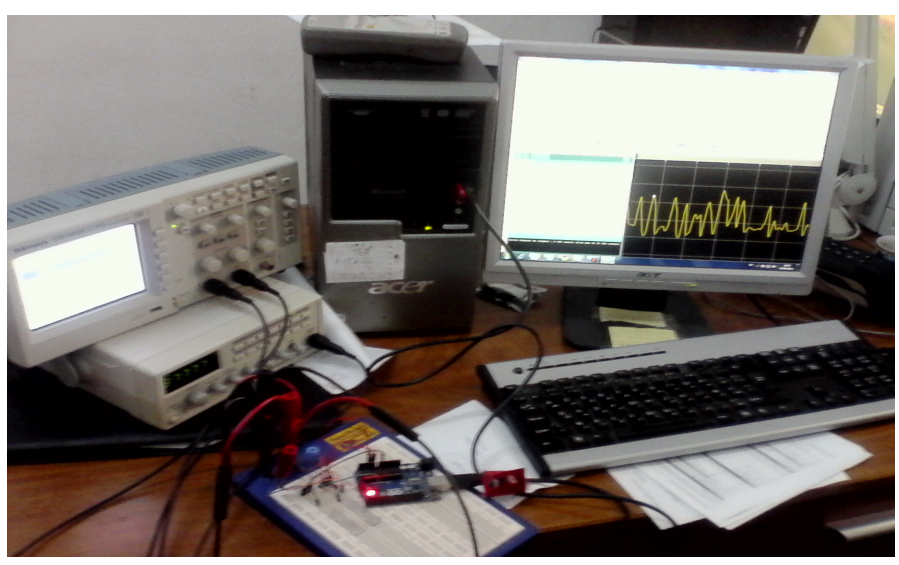

Fig. 1: Diagram of Real Time Implementation

\subsection{ARDUINO I/O interface mode}

The first mode is the one to use the ARDUINO card as an I/O interface with MATLAB Simulink $($. After loading the firmware "adioserv.pde" into the Arduino card, we install Arduino I/O library to Simulink Libraries.

This implementation mode is used as a real-time emulator of robot. The robot model is developed in Simulink using the embedded MATLAB function and then it is transferred to the Arduino device as DSP target [16].

In this phase of implementation, a noise was added to the output of the system. The added signal is a sinusoidal signal with frequency equal to $140 \mathrm{~Hz}$ and amplitude $\pm 10 \%$ of $y$.

First, we present in Figs. 23, respectively, the real $x_{1}-x_{2}$ and its estimates $\left(\hat{x}_{1}-\hat{x}_{2}\right)$.

As shown in Figs. 2 and 3 , the states are very well estimated.

\subsection{ARDUINO Target interface mode}

In this mode of programming, Arduino card becomes a target of the Simulink code compiled with the tool "Run on Target Hardware". The Arduino kit operate completely in an autonomous way. It can also be managed online via the USB port of the PC (External Mode Enable) [16].

In this second phase of implementation, two noises are added. The 


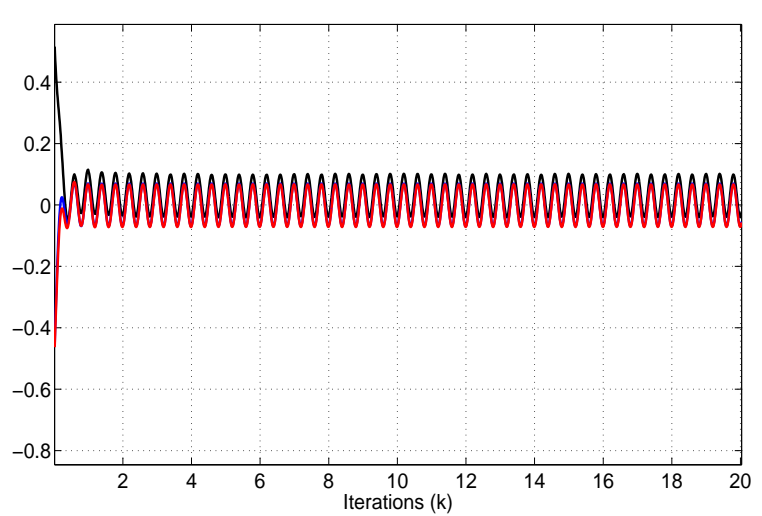

Fig. 2: Response of $x_{1}(k)$ (black line) and its estimates with O.S.L (red line) and DMVT (blue line)

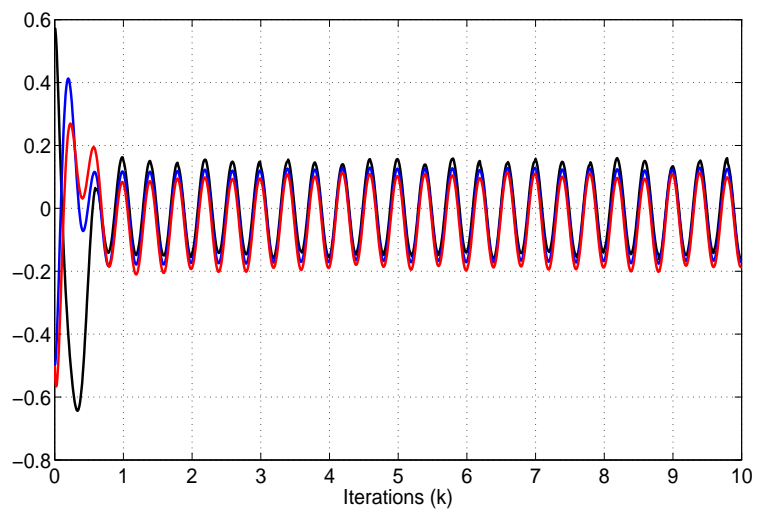

Fig. 3: Response of $x_{2}(k)$ (black line) and its estimates with O.S.L (red line) and DMVT (blue line)

first is applied to the output of the system and the second is introduced on the nonlinear function. The added signals are sinusoidal signals with variable frequencies (between $40 \mathrm{~Hz}$ and $3800 \mathrm{~Hz}$ ) and amplitude $\pm 25 \%$ of $y$ and $f$.

The reconstruction of output signals is provided by the sending of the desired data on the PWM outputs. These PWM outputs are then connected to low pass filters (with $R=3.9 K \Omega$ and $C=33 \mu F$ ).

Note : The choice of low pass filter parameters is based on the frequency of the PWM signal (on most pins of Arduino the frequency is approximately $490 \mathrm{~Hz}$ ).

We present in Figs. 45 respectively the real $x_{2}-x_{3}$ and its estimates $\left(\hat{x}_{2}-\hat{x}_{3}\right)$.

As shown in Figs. 4 5, for a small value of $\lambda(\lambda=3)$, the state is very well estimated using the property of one sided-Lipschitz and also using the DMVT theorem. In the next part, the value of $\lambda$ will be increased to test the performance of the two observers.

Consider now $\lambda=500$, with the same approach, we obtain :

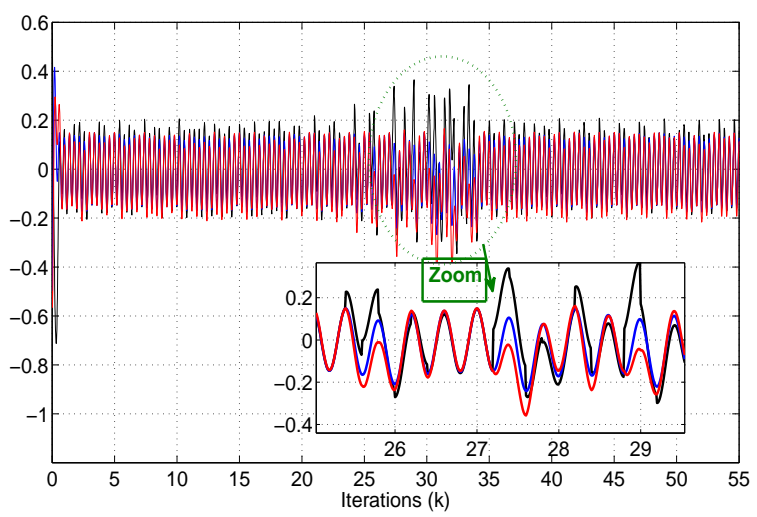

Fig. 4: Response of $x_{2}(k)$ (black line) and its estimates with O.S.L (red line) and DMVT (blue line)

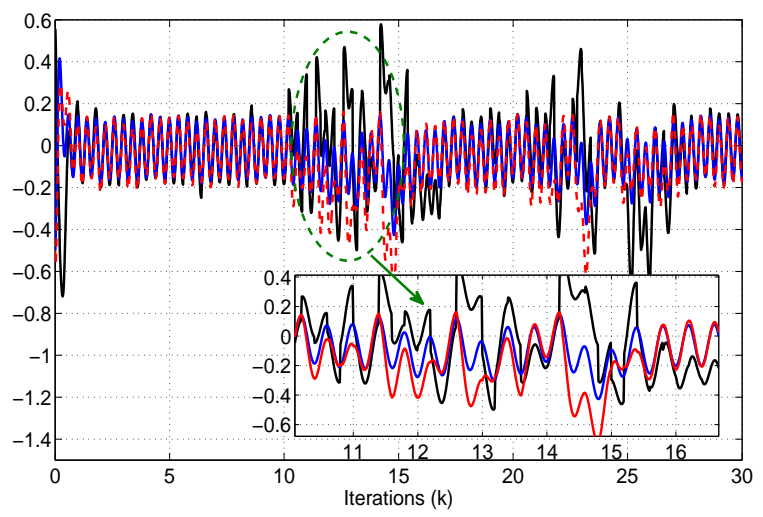

Fig. 5: Response of $x_{3}(k)$ (black line) and its estimates with O.S.L (red line) and DMVT (blue line)

$$
L_{1}=\left[\begin{array}{cc}
0.4610 & 0.01 \\
-0.486 & 0.3662 \\
0 & -0.2006 \\
0.0195 & 0.0462
\end{array}\right] ; L_{2}=\left[\begin{array}{cc}
0.9 & 0.01 \\
-0.486 & 1.1077 \\
0 & 0.6035 \\
0.0195 & 8.6082
\end{array}\right]
$$

The state and its estimates are represented below in Fig. 6 keeping the same initial conditions given previously.

\section{Remarks.}

(1) it is remarkable that the estimated states by the DMVTObserver, compared to those estimated by the One-sidedObserver, are more reliable especially with a very large Lipschitz constant. The results given by O.S.O are biased (Fig 6).

(2) Another advantage is that they are related to the computation time. Indeed, when solving the LMIs, it is quite clear that the DMVT-Observer allocates less memory and computing time compared to the One-sided-Observer (Only three variables are to search with DMVT, while with the One-Sided-Observer more than 12 parameters have to be determined). 


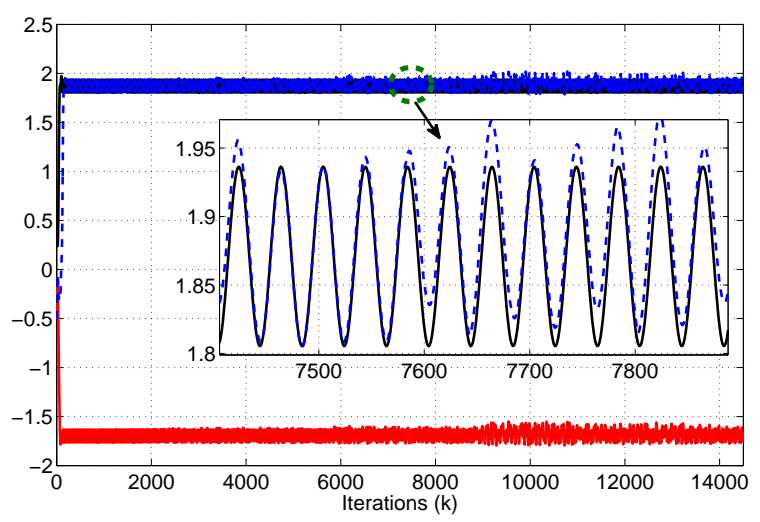

Fig. 6: Response of $x_{1}(k)$ (black line) and its estimates with O.S.L (red line) and DMVT (blue line)

\section{CONCLUSION}

Efficient design of two observers for a class of nonlinear discrete systems are presented.

The two methods are then applied to real time state estimation of the one-link flexible joint robot using ARDUINO MEGA 2560 device in two modes. The results have confirmed the high quality of estimation offered by the DMVT design method with: the presence of noises whose the amplitudes and frequencies are variable and with a large value of Lipschitz constant. The use of the DMVT had ensured a performed stability analysis with non restrictive sufficient condition based on LMI.

\section{Acknowledgment}

The authors would like to thank l'Institut Supérieur des Systèmes Industriels de Gabès and l'Institut Français de Tunise for the realization of the practical tests.

\section{REFERENCES}

[1] Masoud Abbaszadeh and Horacio J. Marquez. Nonlinear observer design for one-sided lipschitz systems. In Proc. American Control Conf., pages 5284-5289, Marriott Waterfront, Baltimore, MD, USA, June 30-July 02, 2010.

[2] A. Alessandri. Design of observers for lipschitz nonlinear systems using lmi. In NOLCOS, IFAC Symposium on Nonlinear Control Systems, Stuttgart, Germany, 2004.

[3] M. Benallouch, M. Boutayeb, and M. Zasadzinski. Observers design for one-sided lipschitz discrete-time systems. Syst. Control Letters, 61:879-886, 2012.

[4] M. Boutayeb. Identification of nonlinear systems in the presence of unknown but bounded disturbances. IEEE Trans. on Autom. Control, 45:1503-1507, 2000.

[5] M. Boutayeb and C. Aubry. A strong tracking extended kalman observer for nonlinear discrete-time systems. IEEE Trans. on Autom. Control, 44:1550-1556, 1999.

[6] S. Boyd, L. El Ghaoui, E. Ferron, and V. Balakrishnan. Linear matrix inequalities in systems and control theory. Studies in Applied Mathematics SIAM, Philadelphia, 15 edition, 1994.
[7] Q.P. Ha and H. Trinh. State and input simultaneous estimation for a class of nonlinear systems. Automatica, 40:1779-1785, 2004.

[8] Pan Jinfeng, Meng Min, and Feng Jun-e. A note on observers design for one-sided lipschitz nonlinear systems. In Control Conference (CCC), 2015 34th Chinese, pages 1003-1007, July 2015.

[9] Linlin Li, Ying Yang, Yong Zhang, and S.X. Ding. Fault estimation of one-sided lipschitz and quasi-one-sided lipschitz systems. In Control Conference (CCC), 2014 33rd Chinese, pages 2574-2579, July 2014.

[10] W. Lin and C. Byrnes. Remarks on linearization of discretetime autonomous systems and nonlinear observer design. Syst. Control Letters, 25:31-40, 1995.

[11] P.R. Pagilla and Y. Zhu. Controller and observer design for lipschitz nonlinear systems. In Proc. American Control Conf., pages 2379-2384, Boston, Massachusetts,USA, 2004.

[12] R. Rajamani. Observer for lipschitz nonlinear systems. IEEE Trans. on Autom. Control, 43:397-401, 1998.

[13] M. Spong. Modeling and control of elastic joint robots. Trans. ASME, J. Dyn. Syst., Meas. Control, 109:310-319, 1987.

[14] A. Thabet, M. Boutayeb, and M. N. Abdelkrim. On the modeling and state estimation for dynamic power system. Int. J. of Electronics Science and Engineering, 7 (2):181-190, 2013.

[15] A. Thabet, M. Boutayeb, and M.N. Abdelkrim. Real time dynamic state estimation for power system. Int J. of Computer Applications, 38-2:11-18, 2012.

[16] A. Thabet, G.B.H Frej, and M. Boutayeb. Observer-based feedback stabilization for lipschitz nonlinear systems with extension to $h \infty$ performance analysis: Design and experimental results. IEEE Trans. on Control Systems Technology, 26 (1):321-328, 2018.

[17] Chunyan Wang, Zongyu Zuo, Zongli Lin, and Zhengtao Ding. Consensus control of a class of lipschitz nonlinear systems with input delay. Circuits and Systems I: Regular Papers, IEEE Transactions on, 62(11):2730-2738, Nov 2015.

[18] A. Zemouch and M. Boutayeb. A unified $\mathcal{H}_{\infty}$ adaptive observer synthesis method for a class of systems with both lipschitz and monotone nonlinearities. Syst. Control Letters, 58:282-288, 2009.

[19] A. Zemouch, M. Boutayeb, and G.I. Bara. Observers for a class of lipschitz systems with extension to $\mathcal{H}_{\infty}$ performance analysis. Syst. Control Letters, 57:18-27, 2008.

[20] F. Zhu and Z.Han. A note on observers for lipschitz nonlinear systems. IEEE Trans. on Autom. Control, 47:1751-1754, 2002. 